\title{
Практические алгоритмы
}

\section{ВЕДЕНИЯ ПАЦИЕНТОВ С КОГНИТИВНЫМИ НАРУШЕНИЯМИ}

\section{B.B. ЗАХАРОВ, Н.В. ВАХНИНА}

Федеральное государственное автономное образовательное учреждение высшего образования «Первый Московский государственный медицинский университет имени И.М. Сеченова» Министерства здравоохранения Российской Федерации (Сеченовский университет): 119991, Россия, г. Москва, ул. Трубецкая, 8, стр. 2

\section{Информация об авторах:}

Захаров Владимир Владимирович - д.м.н., профессор кафедры нервных болезней и нейрохирургии Федерального государственного автономного образовательного учреждения высшего образования «Первый Московский государственный медицинский университет имени
И.М. Сеченова» Министерства здравоохранения Российской Федерации (Сеченовский университет); тел.: +7(499) 248-63-00

Вахнина Наталья Васильевна - К.М.Н., доцент кафедры нервных болезней и нейрохирургии Федерального государственно- го автономного образовательного учреждения высшего образования «Первый Московский государственный медицинский университет имени И.М. Сеченова» Министерства здравоохранения Российской Федерации (Сеченовский университет); тел.: +7(499) 248-63-00

\section{PEЗЮME}

В статье изложены современные подходы к ведению пациентов с когнитивными нарушениями различной этиологии. Приведены алгоритмы синдромальной (легкие, умеренные, выраженные) и нозологической дифференциальной диагностики когнитивных расстройств, показано место диагностических лабораторных, инструментальных и нейрорадиологических методов исследования. Обсуждаются данные последних исследований об эффективности когнитивного тренинга, оптимизации образа жизни, физической активности, профилактическом значении своевременного лечения сердечно-сосудистых и иных заболеваний. Показаны возможности современной нейропротективной и симптоматической терапии когнитивных нарушений различной этиологии, место EGB 761 в лечении заболеваний с когнитивными нарушениями.

Ключевые слова: когнитивные нарушения, деменция, нейропротективная терапия, экстракт гинкго билоба

Для цитирования: Захаров В.В., Вахнина Н.В. Практические алгоритмы ведения пациентов с когнитивными нарушениями. Медицинский совет. 2019; 6: 27-33. DOI: https://doi.org/10.21518/2079-701X-2019-6-27-33.

Конфликт интересов: авторы заявляют об отсутствии конфликта интересов.

\section{Practical algorithms}

\section{FOR MANAGING PATIENTS WITH COGNITIVE IMPAIRMENTS}

\author{
Vladimir V. ZAKHAROV, Natalya V. VAKHNINA \\ Federal State Autonomous Educational Institution of Higher Education Sechenov First Moscow State Medical University of the Ministry of \\ Health of the Russian Federation (Sechenov University): 8, Trubetskaya Street, Moscow, 119991, Russia, p. 2
}

\section{Author credentials:}

Zakharov Vladimir Vladimirovich - Dr. of Sci.(Med.), Professor, Chair for Nervous Diseases and Neurosurgery, Federal State Autonomous Educational Institution of Higher Education «Sechenov First Moscow State Medical University» of the Ministry of
Heath of the Russian Federation (Sechenov University); tel.: +7(499) 248-63-00 Vakhnina Natalya Vasilyevna - Cand. of Sci. (Med.), Associate Professor, Chair for Nervous Diseases and Neurosurgery, Federal State Autonomous Educational
Institution of Higher Education «Sechenov First Moscow State Medical University» of the Ministry of Heath of the Russian Federation (Sechenov University); tel.: +7(499) 248-63-00

\section{ABSTRACT}

The article presents modern approaches to the management of patients with cognitive impairment of different etiology. The authors presented the algorithms for syndromic (mild, moderate, severe) and nosological differential diagnosis of cognitive disorders, and showed the importance of diagnostic laboratory, instrumental and neuroradiological research methods. They discussed the latest research data on the effectiveness of cognitive training, lifestyle optimization, physical activity, and the prophylactic value of timely treatment of cardiovascular and other diseases. The paper shows the possibilities of modern neuroprotective and symptomatic therapy of cognitive impairment of different etiology, the role of EGB 761 in the treatment of diseases with cognitive impairment.

Keywords: cognitive impairment, dementia, neuroprotective therapy, ginkgo biloba extract

For citing: Zakharov V.V., Vakhnina N.V. Practical algorithms for managing patients with cognitive impairments. Meditsinsky Sovet. 2019; 6: 27-33. DOI: https://doi.org/10.21518/2079-701X-2019-6-27-33.

Conflict of interest: The authors declare no conflict of interest. 
амым распространенным видом неврологических расстройств, который встречается как в практике неврологов, так и врачей других специальностей, является нарушение высших мозговых (когнитивных, интеллектуальных) функций. Очень трудно представить себе заболевание головного мозга или церебральное осложнение соматического заболевания, которое не оказывало бы негативного влияния на когнитивные функции. По данным отечественного исследования «ПРОМЕТЕЙ», выполненного под руководством академика Н.Н. Яхно, снижение когнитивных функций выявляется почти у 70\% амбулаторных неврологических пациентов пожилого возраста [1]. Риск когнитивных нарушений (KH) значительно повышается при наличии таких заболеваний, как артериальная гипертензия, сахарный диабет, ожирение, гиперлипидемия и др. [2]. Поэтому необходима врачебная настороженность в отношении КН при работе с пациентами пожилого возраста и/или при наличии в анамнезе сердечно-сосудистых заболеваний и других факторов риска КН.

Когнитивные жалобы. Основанием для клинического исследования когнитивных функций служат жалобы пациента на забывчивость, трудности концентрации, снижение умственной работоспособности или повышенную утомляемость при интеллектуальной работе. Иногда когнитивные жалобы носят завуалированный характер. Так, пациенты с КН нередко сообщают о различных неприятных ощущениях в голове («голова как чугунная», «как пыльным мешком ударили», «тяжелая голова», «несвежая голова», «не моя, другая»), которые мешают сосредоточиться, работать и выполнять привычные дела. С нашей точки зрения, подобные жалобы также следует расценивать как субъективные эквиваленты когнитивных трудностей. Пациент с выраженными КН может совсем не предъявлять никаких жалоб из-за снижения критики или анозогнозии. Источником информации о когнитивном снижении в таких случаях являются родственники пациента или иные близкие люди.

Объективизация и оценка выраженности КН. Для объективизации состояния когнитивных функций пациента применяют нейропсихологические методы исследования. Чаще всего в клинической практике используются Миниисследование умственного состояния (англ.: Mini-Mental State Examination, MMSE [3]) и Монреальский когнитивный тест (МоСа-тест) [4]. Последний более предпочтителен, потому что характеризуется большей чувствительностью: МоСа-тест выявляет КН на более ранних стадиях. Так, по данным авторов теста, его чувствительность (при критерии диагноза - 26 баллов) на стадии легких и умеренных КН составляет 90\%, в то время как чувствительность MMSE - только 18\% [4].

О наличии объективных КН у пациента можно говорить при двух условиях:

- при результатах тестов нижепринятых нормативов (26 баллов для МоСа-теста и 25 баллов для MMSE);

- при анамнестических данных о снижении когнитивных функций по сравнению с преморбидным уровнем (индивидуальной нормой).
Рисунок. Алгоритм синдромальной диагностики КН Figure. Algorithm for syndromic diagnosis of cognitive impairment

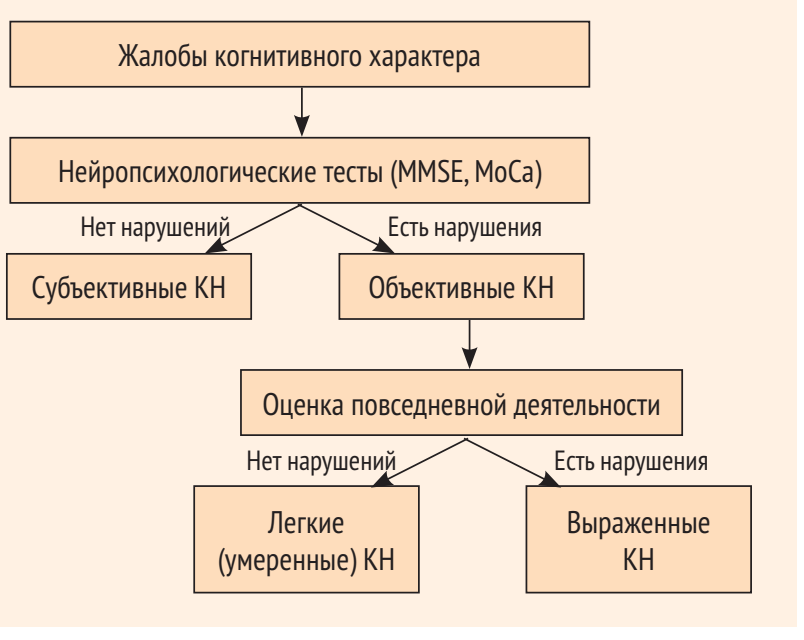

При наличии анамнестических сведений о когнитивном снижении важной задачей врача является определить стадию снижения, так как от этого во многом зависит терапевтическая тактика.

Выделяют три стадии развития когнитивного дефицита: - субъективные КН: имеются жалобы на стойкое снижение когнитивных функций по сравнению с недавним прошлым, однако результаты нейропсихологических тестов (MMSE, МоСа, другие методики) остаются в пределах принятого для соответствующего возраста и уровня образования норматива;

- легкие (умеренные) КН: результаты нейропсихологических тестов (MMSE, MoСа, другие методики) выходят за рамки принятого для возраста и уровня жизни норматива; однако пациенты остаются полностью самостоятельными в повседневной жизни;

- выраженные КН (деменция): когнитивные расстройства выражены настолько, что пациент нуждается в посторонней помощи, по меньшей мере в некоторых обычных жизненных ситуациях (например, при путешествиях, осуществлении финансовых операций, профессиональной деятельности и др.).

Схематически алгоритм диагностики и оценки тяжести КН приведен на рисунке.

Лабораторные и инструментальные исследования при КН. Снижение когнитивных функций не всегда развивается вследствие первичного церебрального заболевания. В некоторых случаях причиной КН может быть церебральная дисфункция вследствие системных дисметаболических расстройств при соматических, эндокринных заболеваниях или экзогенных интоксикациях (табл. 1). В этих случаях своевременная диагностика и коррекция метаболических показателей приводит к полному регрессу или значительному уменьшению выраженности имеющихся расстройств. Поэтому всем пациентам с КН, независимо от их тяжести, рекомендуется лабораторный скрининг дисметаболической энцефалопатии, который включает:

- общий анализ крови; 
- Таблица 1. Дисметаболические энцефалопатии с КН Table 1. Dysmetabolic encephalopathy with cognitive impairment

- Электролитные нарушения (гипо- или гипернатриемия, гипо- или гиперкалиемия, гипо- или гиперкальциемия)

- Сердечная, печеночная, почечная или дыхательная недостаточность

- Алкоголизм, употребление психоактивных веществ, другие интоксикации

- Дефицит витаминов группы В, фолиевой кислоты

- Гипотиреоз, гиперкортицизм, другие эндокринопатии

исследование уровня глюкозы, биохимический скрининг заболеваний печени и почек (АСТ, АЛТ, креатинин, азот мочевины);

- исследование ТТГ, витамина В12, фолиевой кислоты.

У пациентов с быстропрогрессирующими КН необходимо исключать электролитные расстройства, для чего целесообразно исследовать показатели натрия, калия и кальция. Редкой причиной быстропрогрессирующих КН является энцефалопатия Хашимото, которая характеризуется значительным (в десятки раз) повышением уровня антител к тиреопероксидазе.

Нейровизуализация. Желательно, чтобы каждому пациенту с КН любой степени выраженности хотя бы однажды была выполнена нейровизуализация - КТ или МРТ головного мозга. При наличии выраженных или прогрессирующих КН, с нашей точки зрения, нейровизуализация является обязательной. В случае если у пациента в процессе динамического наблюдения существенно изменяются клинические характеристики КН или ускоряется темп их прогрессирования, нейровизуализацию следует выполнить повторно.

Главной задачей нейровизуализации при КН является исключение угрожающих жизни заболеваний, таких как опухоль головного мозга, хроническая субдуральная гематома, абсцесс мозга и др. КТ или МРТ головы позволяют также подтвердить или исключить наличие внутренней гидроцефалии (расширение желудочков головного мозга), что характерно для одной из форм обратимых КН - нормотензивной гидроцефалии. Второй по значимости задачей нейровизуализации является дифференциальный диагноз между первичными церебральными заболеваниями с КН в клинической картине.
Нозологическая диагностика КН. После исключения так называемых «вторичных КН» (дисметаболические нарушения, опухоли головного мозга, нейроинфекция) следует уточнить, какое первичное церебральное заболевание лежит в основе КН. По статистике, чаще всего причиной КН являются болезнь Альцгеймера, сосудистые заболевания головного мозга, лобно-височная дегенерация и диффузная болезнь с тельцами Леви [5]. Нейропсихологические, неврологические и нейрорадиологические дифференциально-диагностические признаки перечисленных заболеваний приведены в таблище 2.

Скрининг и лечение тревожно-депрессивных расстройств. Тревога и депрессия представляют собой самые распространенные в популяции психические расстройства. Они могут вызывать когнитивное снижение или быть коморбидным состоянием, усугубляющим когнитивный дефицит. По эпидемиологическим данным, риск развития депрессии в течение жизни достигает $10 \%$ у мужчин и до $20 \%$ у женщин [6]. Согласно российскому исследованию КОМПАС (2004), распространенность депрессии в общемедицинской практике варьирует от 24 до 64\% [7]. Частота встречаемости расстройств тревожного спектра, по данным популяционных исследований, составляет 6-14\% среди взрослых лиц [8].

Как депрессия, так и тревога оказывает негативное влияние на когнитивные функции. Трехлетнее наблюдение за пациентами с депрессией показало, что во время обострения пациенты испытывают когнитивные трудности в среднем 94\% времени бодрствования. Представляет интерес, что во время ремиссии этот показатель снижается, но остается достаточно высоким (44\%) [9]. Таким образом, у части пациентов когнитивные трудности сохраняются несмотря на регресс эмоциональных расстройств. Возможно, это связано с тем, что депрессия через активацию гипоталамо-гипофизарно-надпочечниковой системы может способствовать атрофическим изменениям в таких важных для познавательной деятельности церебральных структурах, как медиальная височная кора и гиппокамп [10]. Повышенная тревога, по данным Т.Г. Вознесенской,

Таблица 2. Дифференциальный диагноз наиболее частых дементирующих заболеваний

Table 2. Differential diagnosis of the most frequent dementiating diseases

\begin{tabular}{|c|c|c|c|}
\hline Заболевание & Нейропсихологические особенности & $\begin{array}{l}\text { Неврологический } \\
\text { статус }\end{array}$ & Нейрорадиологические характеристики \\
\hline $\begin{array}{l}\text { Болезнь } \\
\text { Альцгеймера }\end{array}$ & $\begin{array}{l}\text { Нарушения памяти преимущественно на события недавнего } \\
\text { прошлого, зрительно-пространственная агнозия, } \\
\text { конструктивная апраксия, акалькулия, аномия }\end{array}$ & Норма & $\begin{array}{l}\text { Атрофия медиальных отделов височных } \\
\text { долей }\end{array}$ \\
\hline $\begin{array}{l}\text { Болезнь диффузных } \\
\text { телец Леви }\end{array}$ & $\begin{array}{l}\text { Брадифрения, флюктуации, умеренные нарушения памяти, } \\
\text { зрительно-пространственного гнозиса, конструктивного } \\
\text { праксиса, управляющих функций, зрительные галлюцинации }\end{array}$ & Паркинсонизм & $\begin{array}{l}\text { Неспецифические атрофические } \\
\text { изменения }\end{array}$ \\
\hline $\begin{array}{l}\text { Хроническая ишемия } \\
\text { мозга }\end{array}$ & $\begin{array}{l}\text { Брадифрения, флюктуации, умеренные нарушения памяти, } \\
\text { зрительно-пространственного гнозиса, конструктивного } \\
\text { праксиса, управляющих функций }\end{array}$ & $\begin{array}{l}\text { Лобная дисбазия, } \\
\text { псевдобульбарный } \\
\text { синдром }\end{array}$ & $\begin{array}{l}\text { Инфаркты мозга, микрокровоизлияния, } \\
\text { диффузные изменения белого вещества } \\
\text { (лейкоареоз) }\end{array}$ \\
\hline $\begin{array}{l}\text { Лобно-височная } \\
\text { дегенерация }\end{array}$ & Нарушение управляющих функций, прогрессирующая афазия & Норма & $\begin{array}{l}\text { Атрофия лобных и передних отделов } \\
\text { височных долей }\end{array}$ \\
\hline
\end{tabular}


также ассоциируется с более низкими результатами нейропсихологических тестов [11].

Учитывая возможное негативное влияние тревоги и депрессии на когнитивные функции и высокую коморбидность тревожно-депрессивных и когнитивных расстройств, у всех пациентов с КН необходимо исследовать эмоционально-поведенческую сферу. Для этого целесообразно использовать формализованные опросники (например, госпитальную шкалу тревоги и депрессии), которые позволяют провести скрининг с минимальными затратами времени врача.

При выявлении тревожно-депрессивных расстройств необходима их медикаментозная коррекция. При этом следует избегать препаратов с холинолитическим эффектом, так как они могут усугублять когнитивный дефект. С учетом безопасности и переносимости предпочтительны антидепрессанты из групп селективных ингибиторов обратного захвата серотонина (эсциталопрам, сертрален, пароксетин, флюоксетин, флювоксамин и др.), ингибиторов обратного захвата серотонина и норадреналина (венлафаксин, дулоксетин, милнаципрам) и другие препараты, не влияющие на ацетилхолинергическую систему (агомелатин, вортиоксетин, тразадон и др.).

Коррекция сосудистых факторов риска и КН. Артериальная гипертензия является одним из наиболее сильных факторов риска КН, что подтверждается многочисленными эпидемиологическими исследованиями. Метаанализ, объединивший 6 крупных международных рандомизированных клинических исследований (Syst-Eur, PROGRESS, SCOPE, SHEP, PRoFESS HYVET, в общей сложности более 19000 пациентов), показал, что на фоне антигипертензивной терапии достоверно снижается риск когнитивного ухудшения. Оно определялось как снижение результата MMSE на 3-4 балла и более. Этот эффект не зависел от класса используемых лекарственных средств. В то же время снижение риска деменции было статистически недостоверным [12]. По ретроспективным наблюдениям прослеживается статистическая связь с возрастом начала терапии и ее продолжительностью: чем моложе были пациенты в начале и чем длительнее было наблюдение, тем более существенно снижался риск деменции [21-25].

Лечение статинами, вероятно, не оказывает значительного влияние на риски возникновения, темпы прогрессирования или выраженность КН. В исследованиях Heart Protection Study [18] и PROSPER [19] не было получено достоверного влияния статинов на когнитивные функции пациентов с сосудистыми факторами риска. В других работах анализировалось влияние статинов на когнитивные функции пациентов с начальными и средними стадиями болезни Альцгеймера. Однако статистически значимых различий с плацебо также не было получено [20].

В двух крупных рандомизированных исследованиях анализировалась связь между терапией дезагрегантами и КН. В работе «Аспирин в лечении бессимптомного атеросклероза» (3350 пациентов в возрасте от 50 до 75 лет) через 5 лет наблюдения отсутствовали достоверные различия по когнитивным показателям между группой ацетилсалициловой кислоты (100 мг/сут) и плацебо [21].
Другие антитромбоцитарные препараты (комбинация ацетилсалициловой кислоты с дипиридамолом медленного высвобождения, клопидогрел) также не показали каких-либо преимуществ перед плацебо (исследование PRoFESS) [22].

Немедикаментозные подходы к профилактике и лечению КН. В последние годы пристальное внимание врачей и исследователей уделяется немедикаментозным стратегиям коррекции КН: физической активности, когнитивному тренингу и правильному питанию. Особенную актуальность немедикаментозные стратегии имеют на начальных стадиях когнитивного дефицита - при синдромах субъективных, легких и умеренных КН.

Влияние физической активности на риск КН и деменции было проанализировано в метаанализе S. Guure и соавт., 2017 [23]. В метаанализ были включены результаты 45 проспективных наблюдений длительностью от 1 до 28 лет, в которых участвовало 117410 пациентов. В результате было получено, что активный в физическом отношении образ жизни ассоциируется со снижением риска деменции на 21-24\%, что статистически значимо. В наибольшей степени физическая активность снижает риск развития болезни Альцгеймера (на 29-38\%), в то время как ее влияние на риск сосудистой деменции оказалось недостоверным. Полученные результаты согласуются с рядом других исследований [24-27]. Однако некоторым авторам не удалось доказать нейропротективного эффекта физической активности в отношении КН. Предполагается, что профилактический эффект физической активности связан со стимуляцией выработки церебральных нейротрофических факторов, что замедляет прогрессирование нейродегенеративного процесса [28-30].

Когнитивный тренинг представляет собой систематические упражнения по тренировке памяти, внимания и других высших мозговых функций, которые проводятся под руководством специалиста (обученного врача, нейропсихолога). Когнитивный тренинг может проводиться индивидуально или в группах, с использованием компьютерных программ или без них. Считается, что когнитивный тренинг, с одной стороны, помогает пациенту адаптироваться и частично преодолеть когнитивный дефект за счет сохранных функций, а с другой стороны, непосредственно влияет на морфофункциональные основы церебральных нейрорепаративных процессов. В систематическом обзоре Buttler М. и соавт, 2018, были проанализированы результаты 11 проспективных исследований когнитивного тренинга у здоровых взрослых лиц и у пациентов с синдромом легких (умеренных) КН. Было показано, что у здоровых лиц когнитивный тренинг достоверно улучшает тренируемую функцию, в то время как у пациентов с легкими или умеренными КН - нет [31]. По данным С.М. Smart et al., 2017, когнитивный тренинг способствует улучшению высших мозговых функций у пациентов с синдромом субъективных КН, при этом величина эффекта когнитивного тренинга в данной популяции пациентов больше, чем других немедикаментозных стратегий [32].

Оптимизация питания также обсуждается в качестве возможной стратегии предотвращения развития или сни- 
жения темпов прогрессирования КН. Многочисленные ретроспективные наблюдения свидетельствуют о возможном благоприятном влиянии витаминов группы B и $\mathrm{E}$, ненасыщенных жирных кислот, ограничения калорийности рациона на когнитивные функции. Однако данные интервенционных проспективных исследований противоречивы и далеко не всегда подтверждают нейропротективный эффект того или иного нутриента [33]. Тем не менее в работе PREDIMED, где 522 пациента с высоким риском сосудистых событий наблюдались в течение 4,8 лет, было показано, что средиземноморская диета с обязательным включением в рацион оливкового масла или орехов способствует улучшению когнитивных функций по MMSE и тесту рисования часов [34]. Одновременно наблюдалось достоверное снижение риска инсульта и других сосудистых событий [35].

Одновременному использованию всех указанных выше немедикаментозных нейропротективных методик было посвящено Финское гериатрическое интервенционное исследование для профилактики развития когнитивных нарушений и нетрудоспособности (Finnish Geriatric Intervention Study to Prevent Cognitive Impairment and Disability, FINGER) [36]. В него было включено 1260 пациентов в возрасте от 60 до 77 лет с повышенным риском развития деменции. Пациенты были рандомизированы на две приблизительно равные по численности группы. В работе с пациентами основной группы в течение двух лет использовались описанные выше методы нелекарственной профилактики, включая консультации с диетологом, регулярные силовые и аэробные физические упражнения, сеансы компьютеризированного когнитивного тренинга. Одновременно осуществлялся контроль сердечно-сосудистых и метаболических факторов риска. В результате было показано, что использование указанных методов способствует достоверному замедлению прогрессирования расстройств внимания и управляющих функций, но мало влияет на память [36].

Медикаментозная терапия КН. Стратегия фармакотерапии КН зависит от стадии и причины когнитивного дефицита, причем в большей степени - от стадии.

При выраженных КН у пациентов с диагнозом болезнь Альцгеймера или хроническая ишемия головного мозга базисную терапию составляют мемантин и/или ингибиторы ацетилхолинэстеразы (донепезил, ривастигмин, галантамин). Их эффективность была неоспоримо доказана в большом числе рандомизированных клинических исследований. На фоне базисной терапии отмечается достоверное улучшение когнитивных функций, частичный регресс поведенческих и психотических расстройств у пациентов с явной деменцией, увеличение времени относительной самостоятельности пациентов и, соответственно, уменьшение затрат времени и труда на уход за данными пациентами. Представляет интерес, что величина терапевтического эффекта базисной терапии больше при болезни Альцгеймера, чем при сосудистой деменции. При недостаточной эффективности выбранного ингибитора ацетилхолинергического препарата целесообразно поменять его на другой препарат из той же фармакологи- ческой группы. Базисную терапию начинают либо с мемантина, либо с ингибитора ацетилхолинэстеразы, а через некоторое время (обычно через 1 - 2 года) при снижении эффективности монотерапии переходят на комбинированную терапию (мемантин + ингибитор ацетилхолинэстеразы) [37-40].

После стабилизации пациента на базисной терапии можно дополнительно использовать нейрометаболические препараты для усиления клинической эффективности (мемоплант, холина альфосцерат, цитиколин и др.). Так, к настоящему времени накоплен большой клинический опыт использования стандартного экстракта гинкго билобы (EGB 761, мемоплант) при деменциях нейродегенеративной и сосудистой этиологии. В рандомизированном контролируемом исследование GINDEM-NP участвовало 400 пациентов старше 50 лет с болезнью Альцгеймера или сосудистой деменцией и с нейропсихиатрическими симптомами (бред, галлюцинации, психомоторное возбуждение и др.). Пациенты получали мемоплант в дозе 240 мг/сут в течение 22 нед. [36]. Было показано, что в группе активной терапии к концу лечения отмечается статистически значимое улучшение как когнитивных функций, так и поведения пациентов. Одновременно наблюдалось также статистически значимое уменьшение выраженности головокружения и шума в ушах [41]. Сходные данные были получены в двух других крупных исследованиях GOTADAY и GOT-IT, в которых участвовали пациенты с БА и сосудистой деменцией [42, 43]. В работе Mazza и соавт. эффективность EGB 761 (240 мг/сут) сравнивалась с донепезилом (5 мг/сут) у пациентов с БА. Было показано, что наибольший регресс как когнитивных, так и других нервно-психических нарушений отмечается на фоне указанных комбинаций двух указанных препаратов, в то время как эффективность монотерапии экстрактом гинкго билобы и донепезилом была сопоставимой [44].

При легких и умеренных $\boldsymbol{К Н ~ т е р а п и е и ̆ ~ п е р в о и ̆ ~ л и н и и ~}$ является нейрометаболические препараты с вероятным нейропротективным эффектом, которые могут не только улучшить когнитивную функцию, но и предотвратить или максимально отсрочить наступление деменции. В связи с этим большой интерес международного научного сообщества вызывают нейропротективные свойства EGB 761 (мемоплант). Действующими веществами мемопланта являются флавоновые гликозиды, терпеновые вещества и гинкголиды, которые оказывают как вазотропный, так и нейрометаболический эффект. Препарат вызывает вазодилатацию преимущественно в пораженных микрососудах (артериолах, капиллярах, венулах), ингибирует фактор активации тромбоцитов, что приводит к уменьшению склеивания и увеличению деформируемости форменных элементов крови [45]. Мемоплант обладает мощным антиоксидантным эффектом $[46,47]$ и защищает нейроны от токсического воздействия альцгеймеровского амилоидного белка $[48,49]$, что делает обоснованным и целесообразным применение мемопланта не только при хронической ишемии головного мозга, но и на ранних стадиях болезни Альцгеймера. 
В российском многоцентровом исследовании [50] 140 пациентов с умеренными КН сосудистой, нейродегенеративной или смешанной этиологии получали EGB 76180 мг 3 раза в день в течение 6 месяцев. Было выявлено статистически значимое улучшение памяти, внимания, управляющих функций (планирование и контроль), номинативной функции речи, а также нормализация эмоционального состояния. Эффективность EGB 761 на ранних и средних стадиях хронической ишемии головного мозга была убедительно показана в большом числе других отечественных и зарубежных исследованиях. При этом важно подчеркнуть, что одновременно с улучшением когнитивных функций наблюдалось уменьшение выраженности других клинических проявлений хронической сосудистой мозговой недостаточности: головной боли, вестибулоатактического синдрома, головокружения, шума в ушах [51-54]. Так, назначение EGB 761 приводило к регрессу головокружения у $50 \%$ пациентов и уменьшению шума в ушах у $90 \%$ больных [55].

Важно подчеркнуть, что терапия EGB 761 не только уменьшает выраженность КН, но и способствует замедлению темпов прогрессирования КН. В рамках многолетних эпидемиологических наблюдений EPIDOS [56] и PAQUID [57] было получено, что на фоне EGB 761 когнитивные функции оставались более стабильными по сравнению с другими сосудистыми и нейрометаболическими препаратами или без терапии.

Исследованию долговременного эффекта EGB 761 посвящена работа С.Л. Тимербаевой и соавт. 34 пациента в возрасте от 45 до 65 лет с диагнозом начальных проявлений недостаточности кровоснабжения головного мозга получали EGB 761 в дозе 120 мг/сут в течение 3 месяцев [53]. Клиническая эффективность исследуемого препарата анализировалась с помощью клинических неврологических, нейропсихологических (тесты на память и внимание) и электрофизиологических методов исследования (количественный анализ ЭЭГ, исследование когнитивного вызванного потенциала Р300). Было показано, что улучшение когнитивных показателей и биоэлектрической активности головного мозга, отмеченное после курса лечения, сохраняется в течение последующих 6-11 месяцев. О сохраняющемся в течение 6-12 месяцев терапевтическом эффекте EGB 761 сообщают также O.B. Зайцева [58] и Н.Л. Кунельская и соавт. [59]. В указанных работах EGB 761 использовался в лечении шума в ушах при нейросенсорной тугоухости [58] и в качестве метаболической поддержки вестибулярной реабилитации у пациентов с головокружением [59]. Таким образом, EGB 761 может использоваться прерывистыми курсами с интервалом в 6-12 месяцев.

Однако в мире имеется также положительный опыт длительного назначения препаратов EGB 761. В проспективном рандомизированном контролируемом исследовании GuideAge 2820 пожилых лиц с легкими или субъективными КН принимали EGB 761 (240 мг/сут) или плацебо в течение 5 лет. Было показано, что длительная терапия (4 года и более) сопровождается достоверным снижением риска развития болезни Альцгеймера [60].

В заключение следует сказать, что проблема КН пока еще далека от своего решения. Необходимы дальнейшие исследования для разработки эффективной терапии БА и других нейродегенеративных заболеваний, которые являются наиболее распространенными причинами КН в популяции. Однако уже сегодня использование индивидуально подобранной терапии, включающей нейропротективные и симптоматические лекарственные средства, позволяет добиться улучшения интеллектуального состояния в большинстве случаев КН как нейродегенеративной, так и сосудистой природы.

Поступила/Received 15.02.2019

\section{ЛИТЕPATYPA/REFERENCES}

1. Захаров В.В. Всероссийская программа исследований эпидемиологии и терапии когнитивных расстройств в пожилом возрасте («Прометей»). Неврологический журнал. 2006;11:27-32. [Zakharov V.V. All-Russian program of study of cognitive disorders epidemiolo gy and therapy in the elderly. («Prometheus») Nevrologichesky Zhurnal. 2006;11:27-32] (In Russ).

2. Kivipelto M., Ngandu T., Laatikainen T. et al. Risk score for the prediction of the dementia risk for 20 years among middle-aged people: a longitudinal, population-based study. Lancet Neurology. 2006;5(9):735-741.

3. Folstein M.F., Folstein S.E., McHugh P.R. MiniMental State: a practical guide for grading the mental state of patients for the clinician. J Psych Res. 1975;12:189-198.

4. Nasreddine Z.S., Philips N.A., Bedirian V. et al. The Montreal cognitive assessment, MoCa: a brief screening tool for mild cognitive impairment. J Am Ger Soc. 2005;53(4):695-699.

5. Яхно Н.Н., Преображенская И.С., Захаров В.В., Степкина Д.А., Локшина А.В., Мхитарян Э.А., Коберская Н.Н., Савушкина И.Ю. Распространенность когпитивных нарушений при неврологических заболеваниях (анализ работы специализированного амбулаторного приема). Неврология, нейропсихиатрия, психосоматика. 2012;2:30-34. [Yakhno N.N., Preobrazhenskaya
I.S., Zakharov V.V., Stepkina D.A., Lokshina A.V., Mkhitaryan E.A., Koberskaya N.N., Savushkina I. Yu. Prevalence of cognitive impairment in neurological diseases (analysis of the specialized outpatient visit). Nevrologiya, Neyropsikhiatriya, Psikhosomatika. 2012; 2: 30-34.] (In Russ)

6. Вейн А.М., Вознесенская Т.Г., Голубев В.Л., Дюкова Г.М. Депрессия в неврологической практике, 3-е изд. М.: МИА. 2007. 208 с. [Wayne AM, Voznesenskaya T.G., Golubev V.L., Dyukova G.M. Depression in Neurological Practice, 3rd Edition. Moscow, MIA. 2007. 208 p.] (In Russ).

7. Оганов Р.Г., Ольбинская Л.И., Смулевич А.Б., Дробижев М.Ю., Шальнова С.А., Погосова Г.В. Депрессии и расстройства депрессивного спектра в общемедицинской практике. Результаты программы КОМПАС. Кардиология. 2004;9:1-8. [Oganov R.G., Olbinskaya L.I., Smulevich A. B., Drobizhev M.Yu., Shalnova S.A., Pogosova G.V. Depression and depressive disorders in general medical practice. Results of the COMPASS program. Kardiologiya. 2004;9:1-8.] (In Russ).

8. The ESEMed/MHEDEA 2000 Investigators. Prevalence of mental disorders in Europe: results from the European study of the epidemiology of mental disorders (ESEMeD) project. Acta Psychiatr Scand. 2004;109(Suppl. 420):21-27.
9. Conradi HJ., Ormel J., de J.P. Presence of individual (residual) symptoms during depressive episodes and periods of remission: a 3-year prospective study. Psychol. Med. 2011:41(6):1165-1174.

10. Sapolsky R.N. Depression, antidepressants and the shrinking of the hippocampus. Natl Acad Sci. 2001;98(22):12320-12322.

11. Вознесенская Т.Г. Акатинол в лечении некогнитивных нервно-психических расстройств при нейрогериатрических заболеваниях. Nevrologiya, Neyropsikhiatriya, Psikhosomatika. 2012;4(2S):1117. [Voznesenskaya T.G. Akatinol in the treatment of non-cognitive neuropsychiatric disorders in neuro-geriatric diseases. Neurology, neuropsychiatry, psychosomatics. 2012;4(2S):11-17.] (In Russ).

12. Ankolekar S., Geeganage C., Anderton et al. Clinical trials for prevention post stroke cognitive impairment. J of Neurological Sciences. 2010;299:168-174.

13. Haag M.D., Hofman A., Koudstaal PJ. et al. Duration of antihypertensive drug use and risk of dementia: a prospective cohort study. Neurology. 2009;72:1727-1234.

14. Perila R., White L.R., Masaki K. et al. Reducing the risk of dementia: efficacy of long term treatment of hypertension. Stroke. 2006;37:1165-1170.

15. Guo Z., Fratiglioni L., Zhu L. et al. Occurrence and progression of dementia in a community population aged 75 years and older: relation- 
ship of antihypertensive medication use. Arch Neurol. 1999:56:991-996.

16. Khachaturian A.S., Zandi P.P., Lyketos C.G. et al. Antihypertensive medication use and incidence of Alzheimer's disease: the Cache County Study. Arch Neurol. 2006;63:686-692.

17. Qui C., von Strauss E., Fastbom J. et al. Low blood pressure and risk of dementia in the Kungholmen study: a 6 years follow-up study. Arch Neurol. 2003;60:223-228.

18. Heart Protection Study Collaborative Group. MRC/BHF Heart Protection Study of cholesterol lowering with simvastatin in 20536 high-risk individuals: a randomized placebo-controlled trial. Lancet. 2002;360:7-22.

19. Shepherd J., Blauw G.J., Murphy M.B., Bollen E.L.E.M., Buckley B.M., Cobbe S.M., et al. Pravastatin in elderly individuals at risk of vascular disease (PROSPER): a randomised controlled trial. Lancet. 2002;360:1623-30.

20. McGuinness B., Craig D., Bullock R., Passmore P. Statins for the treatment of dementia. Cohrane Database Syst Rev. 2014;8(7):CD 007514.

21. Price J.F., Stewart M.C., Deary IJ. et al. Low dose aspirin and cognitive function in middle aged to elderly adults: randomized controlled trial. BMJ. 2008;437:1198.

22. Diener H.C., Sacco R.L., Yusuf S. et al. Prevention Regimen for Effectively Avoiding Second Strokes (PRoFESS) Study Group. Effects of aspirin plus extended-release dipyridamole versus clopidogrel and telmisartan on disability and cognitive function after recurrent stroke in patients with ischaemic stroke in the Prevention Regimen for Effectively Avoiding Second Strokes (PRoFESS) trial: a double-blind, active and placebo controlled study. Lancet Neurol. 2008;7:875-884

23. Guire C.B., Ibrahim N.A., Adam M.B., Said S.M. Impact of physical activity on cognitive decline, dementia and its subtypes: meta-analysis of prospective studies. Biomed Res Int. 2017;2017:9016924

24. Sofi F., Valecchi D., Bacci D., et al. Physical activity and risk of cognitive decline: a meta-analysis of prospective studies. Journal of Internal Medicine. 2011;269(1):107-117

25. Hamer M., Chida Y. Physical activity and risk of neurodegenerative disease: a systematic review of prospective evidence. Psychological Medicine. 2009;39(1):3-11.

26. Blondell S.J., Hammersley-Mather R., Veerman J.L. Does physical activity prevent cognitive decline and dementia?: a systematic review and meta-analysis of longitudinal studies. BMC Public Health. 2014:14:510.

27. Wang H.X., Karp A., Winblad B., Fratiglioni L. Late-life engagement in social and leisure activities is associated with a decreased risk of dementia: a longitudinal study from the Kungsholmen project. American Journal of Epidemiology. 2002;155(12):1081-1087.

28. Kishimoto H., Ohara T., Hata J., et al. The longterm association between physical activity and risk of dementia in the community: the Hisayama Study. European Journal of Epidemiology. 2016;31(3):267-274.

29. Laurin D., Verreault R., Lindsay J., MacPherson K., Rockwood K. Physical activity and risk of cognitive impairment and dementia in elderly persons. Archives of Neurology. 2001;58(3):498-504

30. Morgan G. S., Gallacher J., Bayer A., Fish M. Ebrahim S., Ben-Shlomo Y. Physical activity in middle-age and dementia in later life: findings from a prospective cohort of men in caerphilly, South Wales and a meta-analysis. Journal of Alzheimer's Disease. 2012;31(3):569-580.

31. Butler M., McCreedy E., Nelson V.A., Desai P., Ratner E., Fink H.A., et al. Does Cognitive Training Prevent Cognitive Decline? A Systematic Review. Ann Intern Med. 2018;168:63-68.

32. Smart C.M., Karr J.E., Areshenkoff C.N. et al. NonPharmacologic Interventions for Older Adults with Subjective Cognitive Decline: Systematic Review, Meta-Analysis, and Preliminary Recommendations. Neuropsychol Rev. 2017;27:245-257.

33. Smith PJ., Blumenthal J.A. Dietary factors and cognitive decline. J Prev Alzheimers Dis. 2016;3(1):53-64
34. Martinez-Lapiscina E.H., Clavero P., Toledo E., Estruch R., Salas-Salvado J., San Julian B. Sanchez-Tainta A., Ros E., Valls-Pedret C., Martinez-Gonzalez M.A. Mediterranean diet improves cognition: the PREDIMED-NAVARRA randomised trial. J Neurol Neurosurg Psychiatry. 2013;84(12):1318-25.

35. Estruch R., Ros E., Salas-Salvadó J., Covas M.I., Corella D., Arós F., Gómez-Gracia E., RuizGutiérrez V., Fiol M., Lapetra J., LamuelaRaventos R.M., Serra-Majem L., Pintó X., Basor J., Muñoz M.A., Sorlí J.V., Martínez J.A., MartínezGonzález M.A., PREDIMED Study Investigators. N Engl J Med. 2013;368(14):1279-1290.

36. Ngandu T., Lehtisalo J., Solomon A. et al. A 2 year multidomain intervention of diet, exercise, cognitive training and vascular risk monitoring versus control to prevent cognitive decline in atrisk elderly people (FINGER): a randomized control trial. Lancet. 2015;385(9984):2255-2263.

37. Birks J. Cholinesterase inhibitors for Alzheimer's disease. Cochrane Libr. 2009. doi: 10.1002/14651858.CD001191.

38. Chen Y., Zhang J., Wang Y., Yuan J., Hu W. Efficacy of Cholinesterase Inhibitors in Vascular Dementia: An Updated Meta-Analysis. Eur Neurol. 2016;75:132-141.

39. McShane R., Areosa Sastre A., Minakaran N. Memantine for dementia. Cochrane Database of Systematic Reviews. 2006;2:CD003154.

40. Thomas S.J., Grossberg G.T. Memantine: a review of studies into its safety and efficacy in treating Alzheimer's disease and other dementias. Clinical intervention in aging. 2009;4:367-377.

41. Napryeyenko O., Borzenko I.; GINDEM-NP Study Group. Ginkgo biloba special extract in dementia with neuropsychiatric features. A randomised placebo-controlled, double-blind clinical trial. Arzneimittelforschung. 2007;57(1):4-11.

42. Ihl R. Gingko biloba extract EGb $761{ }^{\circledR}$ : clinical data in dementia. Int Psychogeriatr. 2012;24:35-40.

43. Ihl R., Bachinskaya N., Korczyn A.D., Vakhapova V. Tribanek M., Hoerr R., Napryeyenko O.; GOTADAY Study Group. Efficacy and safety of a once-daily formulation of Ginkgo biloba extract EGb 761 in dementia with neuropsychiatric features: a randomized controlled trial. Int J Geriatr Psychiatry. 2011;26(11):1186-94.

44. Mazza M., Capuano A., Bria P., Mazza S. Ginkgo biloba and donepezil: a comparison in the treatment of Alzheimer's dementia in a randomized placebo-controlled double blind study. Eur $\rfloor$ Neurol. 2006;13(9):981-5.

45. Koltringer P., Langsteger W., Ober O. Dose-dependent hemorheological effects and microcirculatory modifications following intravenous administration of Ginkgo biloba special extract EGb 761. Clinical Hemorheology. 1995;15:649-656.

46. Rosler M., Retz W., Thome J., Riederer P. Free radicals in Alzheimer's dementia: currently available therapeutic strategies. I Neural Transm Suppl. 1998;54:211-219.

47. Abdel-Kader R., Hauptmann S., Keil U., Scherping I., Leuner K., et al. Stabilization of mitochondrial function by Ginkgo biloba extract (EGb 761). Pharmacological Research. 2007;56:493-502.

48. Wu Y., Wu Z., Butko P., Christen Y., Lambert M.P., et al. Amyloid-binduced pathological behaviors are suppressed by Ginkgo biloba extract EGb 761and ginkgolides in transgenic Caenorhabditis elegans. Journal of Neuroscience. 2006;26:13102-13113.

49. Luo Y., Smith J.V., Paramasivam V., Burdick A., Curry K.J., et al. Inhibition of amyloid-beta aggregation and caspase-3 activation by the Ginkgo biloba extract EGb761. Proc Natl Acad Sci U S A. 2002;99(19):12197-12202.

50. Яхно Н.Н., Захаров В.В., Локшина А.Б., Гаврилова С.И., Федорова Я.Б., Густов А.В., Коршунова ЮА. Дзяк ЛА. Мизякина Е.В. Одинак М.М., Емелин А.Ю., Пилипенко П.И., Вострикова Е.В. Танакан (EGb 761) в терапии умеренных когнитивных нарушений (мультицентровое исследование). Журн. неврологии и nсихиатрии. 2006;12:41-6. [Yakhno N.N. Zakharov V.V., Lokshina AB, Gavrilova S.I., Fedorova Ya.B., Gustov A.V., Korshunova Yu.A., Dzyak L.A., Mizyakina E.V., Odinak M.M., Emelin A.Yu., Pilipenko P.I., Vostrikova E.V. Tanakan (EGb
761) in the treatment of moderate cognitive impairment (multicenter study). Zhurn.Nevrologii i Psikhiatrii. 2006;12:41-6.] (In Russ).

51. Шахпаронова Н.В., Кадыков А.С. Место препарата Билобил в комплексной терапии сосудистых заболеваний головного мозга. Ліки Украіни. 2010;9:54-6. [Shakhparonov N.V., Kadykov A.S. Place of Bilobil in the complex therapy of vascular brain diseases. Liki Ukrainy. 2010;9:54-6.] (In Russ).

52. Яхно Н.Н., Суслина 3.А., Грибачева И.А., Одинак М.М., Коберская Н.Н., Мхитарян Э.А. Влияние препарата Билобил форте на умеренные когнитивные нарушения у пациентов с дисциркуляторной энцефалопатией 1-2 стадии. Клиниконейропсихологическое исследование. РМЖ. 2007;16(12):1626-1629. [Yakhno N.N., Suslina Z.A., Gribacheva I.A., Odinak M.M., Koberskaya N.N., Mkhitaryan E.A. Effect of Bilobil Forte on moderate cognitive impairment in patients with stage 1-2 dyscirculatory encephalopathy. Clinical and neuropsychological research. $R M J$. 2007;16(12):1626-1629.] (In Russ).

53. Тимербаева С.Л., Суслина 3.А., Бодарева Э.А., Федин П.А., Корепина О.С., Первозванский Б.Е. Танакан в лечении начальных проявлений недостаточности кровоснабжения мозга: эффективность, переносимость и отдаленные результаты. Журн. неврол. и психиатр.им. С.С. Корсакова. 1999:54-61. [Timerbaeva S.L., Suslina Z.A., Bodareva E.A., Fedin P.A., Korepina OS, Pervozvansky B.E. Tanakan in the treatment of the initial manifestations of cerebral circulation insufficiency: efficacy, tolerability and long-term results. Zhurn. Nevrol. i psykhiatr. im. S.S. Korsakov. 1999:54-61.] (In Russ).

54. Яхно Н.Н., Дамулин И.В., Захаров В.В., Елкин М.Н., Ерохина Л.Г., Стаховская Л.В., Чекнева Н.С., Суслина 3.А., Тимербаева С.Л., Федин П.А., Бодарева Э.А., Скоромец А.А., Сорокоумов В.А., Ивашкин В.Т., Гигорьев Ю.В., Первозванский Б.Е. Применение танакана при начальных стадиях сосудистой мозговой недостаточности: результаты открытого мультицентрового исследования. Неврол. журн. 1998;3:18-22. [Yakhno N.N., Damulin I.V., Zakharov V.V., Elkin M.N., Erokhina L.G., Stakhovskaya L.V., Chekneva N.S., Suslina Z.A., Timerbaeva S.L., Fedin P.A., Bodareva E.A., Skoromets A.A., Sorokoumov V.A., Ivashkin V.T., Gigoryev Yu.V., Pervozvansky B.E. Use of tanakan in the treatment of primary manifestations of cerebral vascular insufficiency: results of an open multicenter study. Nevrol. Zhurn. 1998;3:18-22.] (In Russ).

55. Morgenstern C., Biermann E. The efficacy of Ginkgo biloba Special Extract EG 761 in patients with tinnitus. International J Clin Farmacol Ther. 2002:40:188-97.

56. Andrieu S., Gillette S., Amouyal K., Nourhashemi F., Reynish E., Ousset PJ., Albarede J.L., Vellas B., Grandjean H.; EPIDOS study. Association of Alzheimer's disease onset with ginkgo biloba and other symptomatic cognitive treatments in a population of women aged 75 years and older from the EPIDOS study. J Gerontol A Biol Sci Med Sci. 2003;58(4):372-7.

57. Amieva H., Meillon C., Helmer C. et al. Gingko biloba extract and long term cognitive decline: a 20 years follow up population based study. PLoS One. 2013:8(1):e52755.

58. Зайцева О.В. Роль танакана в повышении эффективности вестибулярной реабилитации Вестник отоларингологии. 2014;5:66-68. [Zaitseva O.V. The role of tanakan in enhancing the effectiveness of vestibular rehabilitation. Vestnik Otolaringologii. 2014;5:66-68.] (In Russ).

59. Кунельская НЛ., Левина Ю.В., Красюк А.А., Доронина О.М. Оценка эффективности и переносимости танакана при лечении нейросенсорной тугоухости и ушного шума. Вестник отоларингологии. 2009;2:40-42. [Kunelskaya N.L., Levina Yu.V., Krasyuk A.A., Doronina O.M. Evaluation of the efficacy and tolerability of tanakan in the treatment of sensorineural hearing loss and ear noise. Vestnik Otolaringologii. 2009·2:40-42] (In Russ).

60. Vellas B., Coley N., Ousset P.., Berrut G., Dartigues J.F., et al. Long-term use of standardised ginkgo biloba extract for the prevention of Alzheimer's disease (GuidAge): a randomised placebo-controlled trial. Lancet Neurol. 2012;11(10):851-859. 